\title{
Powering Flexible Manufacturing Systems with Intelligent Contact-less Power Transfer
}

\author{
M. Budhia, V. Vyatkin Senior Member IEEE and G. A. Covic Senior Member IEEE \\ Department of Electrical and Computer Engineering \\ The University of Auckland, Auckland 1142, New Zealand
}

\begin{abstract}
Flexible manufacturing systems (FMS) and reconfigurable manufacturing systems (RMS) have both recently evolved to meet increasing demands for flexible manufacturing. This paper investigates the possibility of contact-less power and wireless data transfer to increase the flexibility and safety of various FMS modules by eliminating the need for power or data connections. The paper discusses advantages and implementation aspects of the proposed inductive power transfer (IPT) system and feasibility tests are undertaken to ensure the compatibility of IPT and the FMS modules, which form part of a lab-size modular test bed. In order to increase the cost effectiveness of the design, each module will run for an eight hour shift using a battery which is then automatically recharged via IPT. An energy aware custom switch mode charger has been designed and tested. A greedy heuristic (IEC 61499 compliant) scheduler is implemented to optimize the production schedule based on the level of charge. Design requirements for the charger, energy monitor and scheduler are discussed followed by performance results. The IPT system has been specified however it is still to be incorporated in to the final system.
\end{abstract}

\section{INTRODUCTION}

\section{A. Paper Overview}

This paper describes an investigation into the feasibility of implementing an intelligent contact-less power transfer system for a Flexible Manufacturing System (FMS) module. Combining both contact-less power and wireless communications in an FMS module should allow it to be completely standalone and therefore freely movable, which is intuitively a significant advantage over conventional wired systems.

The reported research project focused on a complete investigation of intelligent wireless power transfer which inherently encompasses a wide range of electrical engineering fields, such as power electronics, industrial informatics and engineering science.

In order to meet the research aim, five research and implementation objectives were defined. These were, investigate wireless power transfer techniques, develop design specifications for a wireless system, make the module energy aware, implement the solutions on the module and consider safety issues.

Energy awareness provides the module with intelligence since it is able to alter its behavior according to the electrical energy status which varies according to the position and workload of the module. If there is a large amount of energy available, the module will carry out energy intensive operations on particular products. Conversely, if there is little energy remaining, total production quota will still be met since the station will only carry out operations on products which require little energy.

The intelligent wireless power transfer research and experimentation was conducted on a Processing station (Fig.1) which forms part of the FESTO Didactic FMS test bed.

The processing station consists of a rotating index table, checker, drill, pusher and controller. The checker checks characteristics of the workpiece, the drill machines it if necessary, while the pusher pushes the workpiece onto the next station. The work pieces are cylindrical plastic objects and are there purely for didactic reasons. The test bed consists of various other modules, each of which can carry out a specific task. Some modules are simple such as the diverting loops which are simply conveyors with controllers while some are significantly more complex such as the material handling station. The test bed is shown in Fig. 2 with the modules surrounding a central conveyor.

The paper is structured as follows. A brief description of flexible manufacturing systems and the IEC 61499 standard is given in part I.B. The motivation for a completely contact-less power transfer system with wireless communications will be elaborated upon in part I.C. The design requirements and methodologies for such a unique system are explained in section II. Results from the implemented system are then presented in section III, followed by the conclusions.

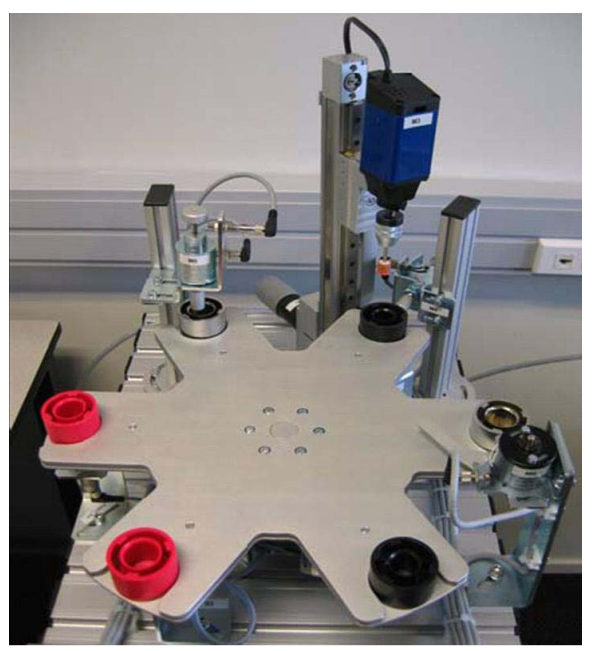

Fig. 1. Processing Station used in our research. 


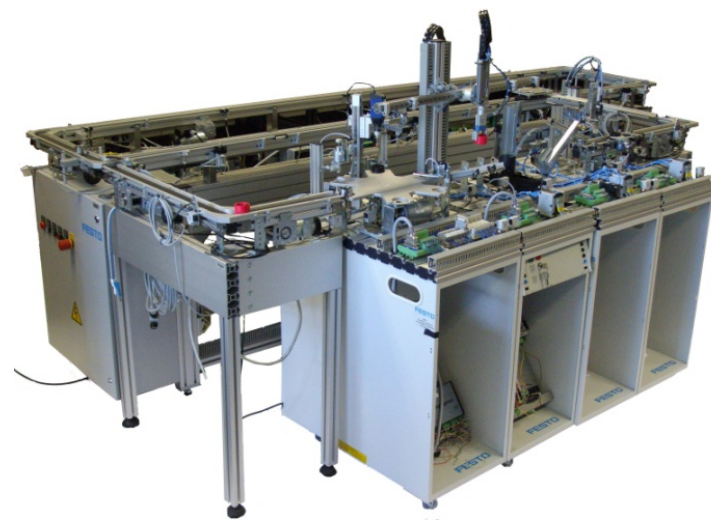

Fig. 2. Model of a flexible reconfigurable modular manufacturing system.

\section{B. Flexible Manufacturing Systems and IEC 61499}

FMS can be comprised of reconfigurable modules which can be placed in different orders to produce a variety of products quickly and efficiently. This enables manufacturers to be extremely agile and hence more competitive. Agility is achieved by having a short time to market, operating with the lowest total cost and by producing higher quality products.

Production schedules with different products can be easily realized since there is no costly and time inefficient retooling. Production can be dynamically altered to match demand and existing parts can be highly customized thus are appealing to a larger range of customers. The major drawbacks of FMS are the high initial cost, the large planning requirement and the need for more complex machines [1].

The IEC 61499 standard defines an event driven architecture that has been specially designed for distributed systems [2, 3]. The standard allows software and hence intellectual property to be encapsulated and distributed. IEC 61499 is directly executable on compatible platforms allowing it to be used in real applications rather than just for modeling purposes. A Functional Block Development Kit (FBDK) is used to create IEC 61499 compliant systems. The basic unit of this standard is an event driven reusable software component called a Function Block (FB).

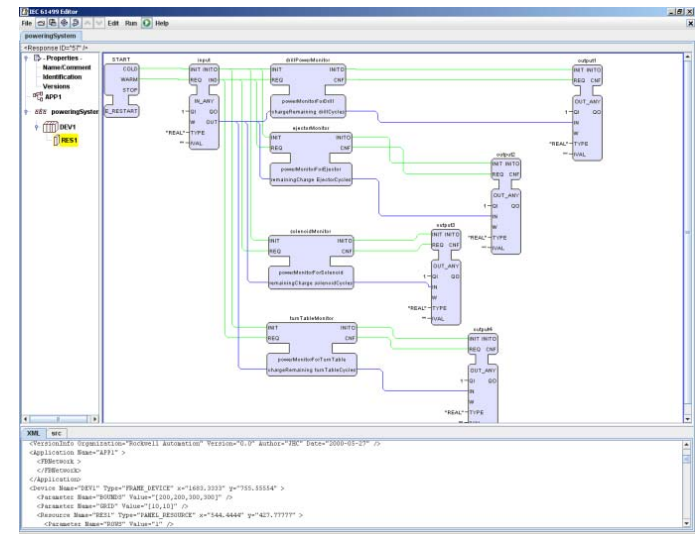

Fig. 3. Function Blocks in FBDK.
A network of them is shown in Fig. 3. Functional blocks contain state machines or software algorithms or both. They are able to be run on any machine which supports Java

Virtual Machine (JVM) which the Mo'Intelligence controller on the processing station does.

\section{Motivation for Wireless Station Modules}

The motivation for wireless modules is best shown with some sample scenarios of reconfiguration which is common in FMS. For example, Fig. 4 shows two possible system arrangements with processing and manipulator stations standing on opposite sides with respect to each other. Current FMS's have multiple cables for each module for power and data. The cables need to be unplugged, moved safely then reconnected which creates a significant time overhead. There may also be safety issues associated with removing cables in certain environments since live parts are exposed. The piece of equipment also needs to be shut down appropriately.

A wireless manufacturing system clearly has numerous advantages over conventional fixed wired system. Wireless power transfer systems are electrically isolated from the manufacturing environment thus do not present any safety risks. There are absolutely no moving parts or electrical contacts to wear out which eliminates maintenance costs. The systems have been proven reliable and are dirt, chemical and moisture resistant $[4,5]$. The system flexibility is increased since modules can be rapidly rearranged with minimum downtime.

\section{REQUIREMENTS AND IMPLEMENTATION OF IPT}

The requirements that need to be satisfied for a wireless power transfer and communication system for the Processing station are described in a methodical manner in the following sub sections. Firstly, the wireless power transfer technique is explained and tested to determine whether it is suitable for the FESTO system. The various inductive power transfer (IPT) options are presented and compared. Following this, the power requirement for the processing station is established based on justified assumptions. Finally the battery charger and energy awareness approaches are described.

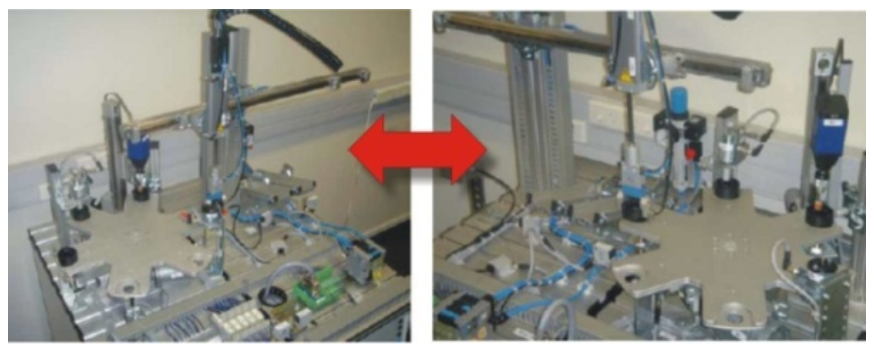

Fig. 4. Trading places of stations in a flexible manufacturing process. 


\section{A. A Contact-less Power Transfer System}

IPT is a well proven wireless power transfer technique which is intended here to power to the modules [4-8]. IPT is based on Ampere's and Faraday's laws which state that an electric current flowing in a an extended loop of wire (track) will produce a magnetic field and that this magnetic field will induce a voltage in a coil of wire (pick-up) when it is placed in proximity with the track. A typical IPT system, as shown in Fig. 5, consists of a resonant power supply which converts the input AC into a high frequency sinusoidal track current at VLF frequencies (typically $10 \mathrm{kHz}-40 \mathrm{kHz}$,). The compensation capacitor is here shown to be in parallel with the track, and helps minimize the VA rating of the supply for a given power demand. An increase in either the operational frequency or current will improve the power transfer to the pick-up, but such increases are limited by component ratings, available switching rates, cost and the track losses. The latter is improved by using Litz wire which reduces the track resistance and minimizes proximity effects $[4,5]$.

The pick-up inductance is comprised of a coil on a ferrite core. This inductor is also tuned for resonance at the track frequency using either a series or parallel compensation capacitor to boost the power transfer. A controller is required to produce a regulated DC output to the load. Typical IPT systems have efficiencies of between $80-90 \%$ and are extremely reliable.

\section{B. IPT Feasibility and Safety Tests}

The majority of industrial IPT power supplies operate at fixed frequency, equal to the resonant frequency of the track inductor and its matching compensation capacitor [6]. Ideally the track inductance should not change significantly during operation otherwise the VA rating of the supply will necessarily increase for a given output power. As the number of FMS modules that may be in close proximity could vary during operation, two simple tests were undertaken representing worst case scenarios. Firstly, the track was laid out without any modules, as shown in Fig. 6 . The track was disconnected from the supply and its inductance was measured using an LCR meter. All of the available FMS modules were then moved over the track and no significant change in inductance was noted. Consequently it was determined that module redeployment would have little operational effect on the supply.

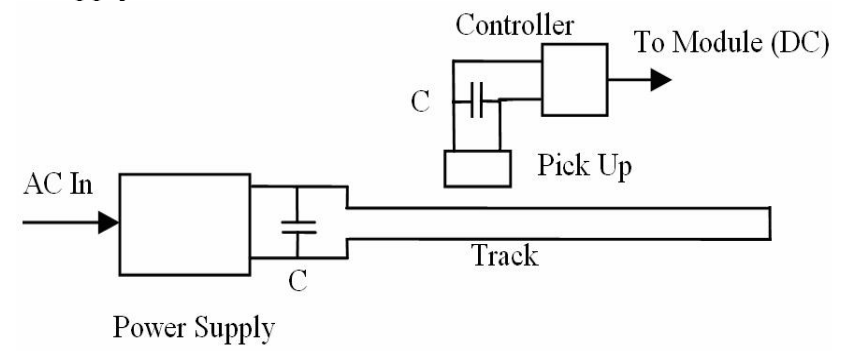

Fig. 5. A Simplified IPT System.
A second concern was to determine if the steel bases of the modules were sufficiently close to act as an inductive heating load since their orientation and proximity to the track can vary. Such a load is undesirable because it increases the standing losses of the supply and could create a heat hazard in a module. To test this possibility, a $1 \mathrm{~kW}$ IPT power supply was used to energize the track and the input power to the supply was measured with and without the modules. No appreciable increase in power demand was measured. As such it was determined that it is possible to transfer power to the FMS modules using IPT with no adverse effects on either the supply or the modules.

\section{Envisaged Implementation}

Following the above tests it was determined that the track should be embedded in the floor such that it encircles the central conveyor (Fig. 6). This allows the surrounding modules to be moved to any position along the track [6]. An alternative charging pad arrangement was considered as described in [7], but this only allows power to be transferred at discrete locations and would significantly limit operational flexibility.

There are several possible track configurations with various levels of flexibility and cost. Here the flexibility of each configuration is defined and compared in terms of their tolerance to horizontal misalignment between the pick-up and centre of the track for a given power transfer.

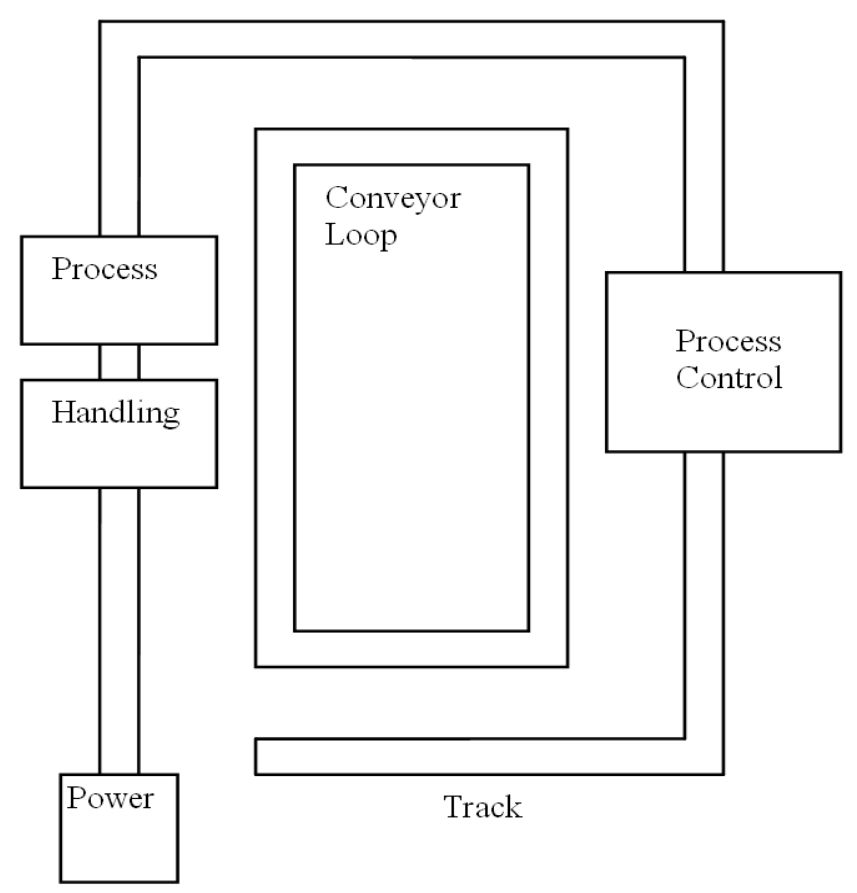

Fig. 6. IPT Track Placement 


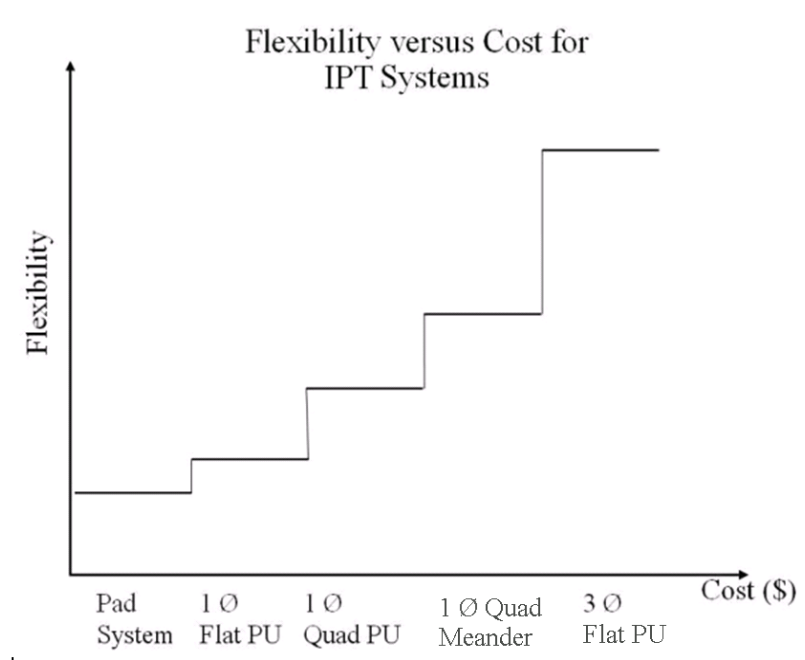

Fig. 7. Flexibility and Cost Relationship for IPT systems $[\varnothing=$ Phase $]$

More flexible systems will enable modules to be placed with less precision. Possible track configurations are: a single phase track with two conductors, a three phase track with six conductors, and a meander track consisting of a repeated single phase track (equivalently having four conductors). In general, increasing the number of conductors improves flexibility without excessive power loss but at increasing cost [6].

The magnetic structure of the pick-up in an IPT system where the load can move relative to the track is almost always flat. The majority of flat pickups simply consist of a single coil but recently a new arrangement using two coils (called the quadrature pickup) has been proposed to improve the horizontal tolerance of a flat pick-up [8]. The graph in Fig. 7 gives a comparison between flexibility and cost of each system.

\section{Processing Station Power Requirement}

Due to cost efficiency reasons (as detailed later) it was decided that a battery should be used to supply the station for an eight hour shift at full duty during a manufacturing shift. The IPT track will then only be energized as required for recharging purposes. An eight hour shift meets the operating requirements for the scope of this investigation. If desired, continuous production shifts could be realized but this would require the IPT track to be energized for longer periods of time. In such a case, the battery is still required to ensure cost effectiveness of the design, since an IPT pick-up rated to supply the average power for module plus battery losses is more economical than one which has the capacity to supply the peak power of $108 \mathrm{~W}$ on demand (24VDC at $4.5 \mathrm{~A}$ ). The measured module power requirement is 160 watt hours or a battery capacity of $6.7 \mathrm{Ah}(24 \mathrm{~V})$. The most commonly used battery chemistries are Lead Acid, Lithium Ion, Nickel Metal Hydride and Nickel Cadmium [9]. The results of the battery investigation are shown in table 1 .
TABLE I

COMPARISON OF BATTERY TYPES $[9,10]$

\begin{tabular}{|c|c|c|c|c|}
\hline \multicolumn{5}{|c|}{ Battery Characteristic Comparison Table } \\
\hline \multirow[t]{2}{*}{ Characteristics } & \multicolumn{4}{|c|}{ Battery Type } \\
\hline & $\mathrm{Ni}-\mathrm{Cd}$ & Ni-MH & $\mathrm{Pb}-\mathrm{Acid}$ & Li-Ion \\
\hline Energy Density $(\mathrm{Wh} / \mathrm{kg})$ & $45-80$ & $60-120$ & $30-50$ & $110-160$ \\
\hline Cycle Life & 1500 & $300-500$ & $200-300$ & $500-1000$ \\
\hline Fast Charge Time (h) & 1 & 3 & 8 & 3 \\
\hline Cell Voltage (V) & 1.25 & 1.25 & 2 & 3.6 \\
\hline \multicolumn{5}{|l|}{ Load Current (A) } \\
\hline Peak (*Capacity) & 20 & 5 & 5 & 2 \\
\hline Best (*Capacity) & 1 & 0.5 & 0.2 & 1 \\
\hline Size & Medium & Medium & Large & Small \\
\hline Efficiency & Good & Good & Poor & Poor \\
\hline Cost (\$) Equiv Capacity & 160 & 190 & 80 & 320 \\
\hline
\end{tabular}

A lead acid battery has been selected since it has the lowest cost to performance ratio. Neither size nor weight are of concern since the processing station has ample space and is on castors.

The points considered when selecting the battery capacity were: The battery voltage drops significantly as the useable capacity is exhausted [9]. The discharge rate affects the battery capacity, the higher the discharge current, the lower the usable capacity. The stated battery capacity is available only if a discharge rate of $\mathrm{C} / 20$ is used, $\mathrm{C}$ being the battery capacity.

The Processing station uses the pulsed discharge mode. High discharge currents are present for short times only while the battery is lightly discharged for a majority of the time. Lead acid cells have short cyclic lives if they are deeply discharged [10]. Accounting for the stated factors and the available manufactured battery capacities, two $12 \mathrm{~V} 7 \mathrm{Ah}$ batteries were selected.

\section{E. Battery Charger Requirements and Implementation}

In order to charge a lead acid battery to full capacity and to prolong its life, the charging process must be done in two stages. Initially, the battery should be charged with constant current and when the voltage reaches a threshold, the charger should switch to constant voltage mode. When the cells reach the end of charge threshold, the charging process should be terminated [10], as shown graphically in Fig. 8.

Cells within the battery can fail to either open or short circuit which presents a safety hazard. A short circuited cell will give off explosive hydrogen gas if charging is attempted. To prevent this, the battery should be tested prior to charging. This involves measuring the charge current and cell voltage and aborting the charging process if a failed cell is detected.

A switch-mode battery charger was chosen because of its efficiency improvement over an equivalent linear regulator type charger [11, 12]. Additionally this eases the design requirements and cost of the IPT system. 


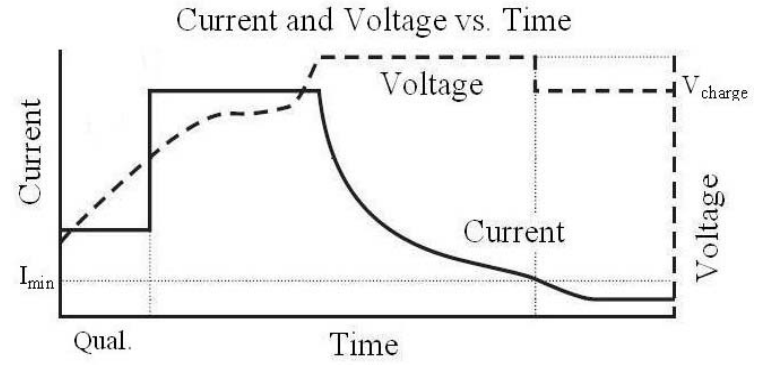

Fig. 8. Optimal Battery Charging Voltage and Current Curves

The chosen parallel tuned IPT pick-up acts as a current source and as such naturally ensures current limiting [4]. A buck topology is used to step down the output voltage of the IPT pick-up and regulate the voltage across and current into the battery. A simplified buck converter is shown in Fig. 9. A pulse width modulated signal from the controller drives the switch whose duty cycle determines the output power. The inductor, capacitor and diode effectively form a filter to smooth the output voltage pulses.

The correct fast charge algorithm is achieved with an application specific integrated circuit controller from Texas Instruments [13]. Battery faults such as cell failure and over temperature are communicated to the station controller via an isolated interface. An integrated safety timer will turn off the charger eight hours after it is switched on. This prevents the battery being overcharged if there is a fault in the controller.

\section{SOFTWARE IMPLEMENTATION OF ENERGY AWARENESS AND ENERGY MONITORING}

As the station runs from a battery during a production shift, an energy aware scheduler is required to meet the desired production quota. Production schedules will be dynamically altered according to the energy remaining in the battery and a command issued to recharge the battery when its capacity reaches a critically low level. For the scope of the project, it has been assumed that there are three types of components being produced.

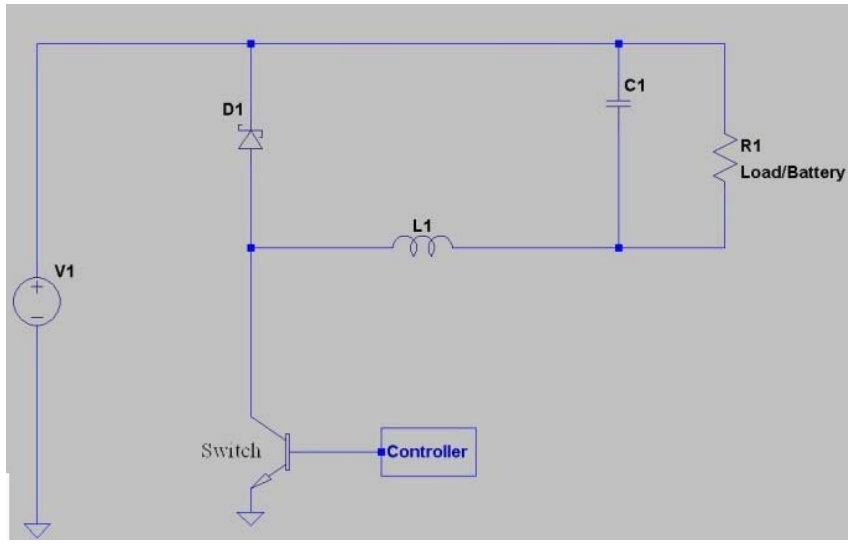

Fig. 9. A Simplified Buck Converter
Each component has a unique energy requirement due to its different associated actions. For example, a component may require more drilling which uses more energy.

The scheduler is a Greedy Heuristic type which looks for local optimums. A solution is built in increments by adding only elements which have least effect on the total outcome [14]. That is, products are only added which require the least energy. A Function Block Development Kit (FBDK) was used to implement the scheduler, based on the IEC 61499 standard.

The station controller requires knowledge of the remaining charge in the battery in real time in order to make production decisions on the fly. The State of Charge (SOC) and State of Health (SOH) need to be monitored accurately. A hybrid monitoring approach has been investigated which combines both voltage and current monitoring $[15,16]$. The SOC is obtained by integrating the current flowing in and out of the battery. The minimum voltage level is reached when the battery capacity is considered exhausted. This minimum is load dependent in that lower values are permissible under high loads (such as the drill). The $\mathrm{SOH}$ is monitored, taking into account the battery capacity, while the total capacity is adjusted each time the battery is charged. The energy monitor has been implemented with a microcontroller which meets the timing requirements and has a sufficient resolution in its Analogue to Digital Converter (ADC). The ADC signal is conditioned using analogue circuitry rather than via software averaging in order to reduce the response time of the microcontroller. This results in more accurate charge monitoring.

\section{A. Safety Issues}

A safety concern relevant to the application of IPT is the possibility of induction heating of metallic objects placed on or very near the track. If such objects were carried by a person working at floor level this is also to be avoided.

An active safety device based on a modulated infra-red (IR) light beam has been developed to increase the systems safety. The beam runs parallel to the track and is active when the track is on. If the beam is broken, an alarm sounds and a signal is sent to the controller to turn off the IPT track supply.

\section{RESULTS}

A complete energy awareness and battery charger prototype system has been constructed and tested over numerous operating cycles. The charger achieves an efficiency of $84 \%$, the input power is $60 \mathrm{~W}$, at $40 \mathrm{~V}$. The battery takes 6 hours to recharge. The IPT pickup and regulator have not yet been incorporated into the final system but are designed to supply the average power demand $(60 \mathrm{~W}$ at $40 \mathrm{~V})$, which is considerably less than the $108 \mathrm{~W}$ peak power required by the station. This significantly reduces the pick-up cost.

The energy monitor has been tested and is able to accurately resolve the remaining battery charge into eight levels while accounting for battery aging. 


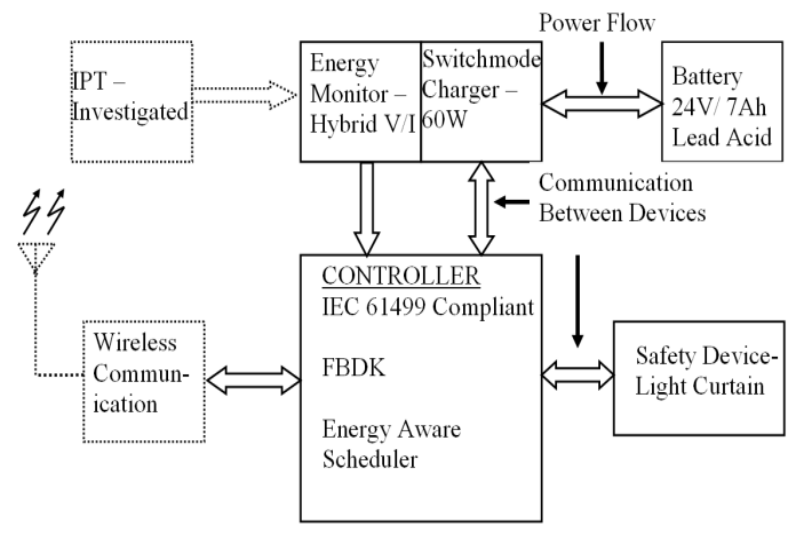

Fig. 10. A Complete System Overview

This resolution was limited in the prototype because the microcontroller had but three remaining digital inputs.

The scheduler correctly simulates in the FBDK by showing the optimum production quota for the three products in a text box, but has yet to be implemented on a wireless IEC 61499 compliant controller. When the remaining battery charge is low, the controller issues a charge command which switches on the charger. A complete system overview is shown in Fig.10. The implemented components are shown with solid lines.

\section{CONCLUSIONS}

An intelligent contact-less power transfer system for a FMS has been scoped and discussed. IPT and wireless data transfer enable increased flexibility and reliability in a FMS since rapid reconfiguration is possible which increases manufacturer agility. They also enable improved safety with lower maintenance costs since there are no mechanical connections. Tests carried out on a processing station have proven that IPT is a completely feasible option.

The design requirements for the battery, charger and energy awareness system have been explained. The processing station will run for an eight hour shift from a battery which will be recharged using IPT. A custom switchmode lead acid battery charger that is fully controllable by the station controller was designed, implemented and tested. Energy awareness is provided by a hybrid voltage-current state of charge monitor which has been implemented with analogue signal conditioning and a microcontroller. The remaining charge is resolved into eight levels and communicated via an insolated interface to the controller. A greedy heuristic scheduler provides the processing station with intelligence allowing the production quota to be changed according to the remaining battery SOC.

\section{REFERENCES}

[1] Lee-Post, Anita, Flexible Manufacturing Systems, University of Kentucky, 2004. Retrieved $3^{\text {rd }}$ April 2007 from http://www.uky.edu/ dsianita/611/fms.html

[2] Vyatkin, V. IEC 61499 Function Blocks for Embedded and Distributed Control Systems design, ISA-o3neida, USA, 2007

[3] IEC 61499, Part 1 "Architecture": Function Blocks for Industrial Process Measurement and Control Systems, Standard, International Electrotechnical Commission, Geneva, 2005

[4] Boys, J.T., Covic, G.A. and Green, A.W. (2000) "Stability and control of inductively coupled power transfer systems, IEE Proc.-Electr. Power Appl., Vol. 147, pp 37 - 43.

[5] Wang, C-S., Covic, G.A. and Stielau, O.H. (2004) "Investigating an LCL Load Resonant Inverter for Inductive Power Transfer Applications", IEEE Trans. on Pwr. Elect., Vol. 19, No. 4, pp 995- 1002

[6] Covic G.A., Boys J.T., Kissin M., and Lu H. (2007) ““”A threephase inductive power transfer system for roadway power vehicles" IEEE Trans., Indus. Elect. Society, 54 no 6, pp. 33703378.

[7] Covic, G. A., Elliott, G., Stielau, O. H., Green R. M. and Boys J. T. (2000) "The design of a contact-less energy transfer system for a people mover system", Int. Conf. on Pwr. Sys. Tech., IEEE-PES / CSEE Powercon 2000, 2, Perth Australia, 4-7 Dec., pp.79-84

[8] Raabe S, Covic G.A and Boys J.T. (2007) "A Quadrature Pickup for Inductive Power Transfer Systems" $2^{\text {nd }}$ IEEE Conf. on Indus. Elect. and Appl, ICIEA 2007, Harbin China 23-25 th May, pp. 68-73

[9] Linden, D. (1995) Handbook of Batteries. Second edition. New York, R. R. Donnelly and Sons Company.

[10] Buchmann, I. (2001) Batteries in a Portable World. Retrieved $8^{\text {th }}$ March 2007 from http://www.buchmann.ca/default.asp

[11] Texas Instruments Incorporated (2007). Switch Mode Lead Acid Battery Charger with user-selectable Charge Algorithms. Retrieved on $18^{\text {th }}$ April from http://focus.ti.com/docs/prod/folders/print/bq2031.html

[12] Cadirci, Y., Ozkazanc, Y. "Microcontroller-based on-line stateof-charge estimator for lead-acid batteries", Journal of Power Sources, 129 (2004)

[13] Texas Instruments Incorporated (2007). Switch Mode Lead Acid Battery Charger with user-selectable Charge Algorithms. Retrieved on $18^{\text {th }}$ April

[14] Caramia, M, Dell'Olmo, P. Effective Resource Management in Manufacturing Systems, Springer,-Verlag, London, 2006

[15] Barsukov, Y., "Challenges and Solutions in Battery Fuel Gauging", Workbook 1-9, Texas Instruments Incorporated (2004

[16] Panasonic VRLA Datasheet - LC-127R2P, Panasonic Corporation, (2005) 